Herman Lombaerts

Catholic University of Leuven, Belgium

\title{
Elżbieta Osewska and Józef Stala (eds.), Religious Education / Catechesis in the Family: A European Perspective
}

\section{Warszawa, Wydawnictwo Universytetu Kardynala Stefana Wyszynskiego, 2010, 277 p., ISBN 978-83-7072-638-6}

Ce livre répond à plusieurs objectifs: revaloriser l'initiation chrétienne au sein de la famille dans l'Europe réintégrée; expliciter les visées, les études et les pratiques d'une éducation religieuse en famille dans chaque pays représenté dans cette étude; permettre le dialogue entre les différents pays et stimuler un rapprochement pour amorcer une collaboration plus directe.

L'introduction comprend trois études d'orientation globale. Un premier texte, « la famille chrétienne moderne comme le premier environnement d'une éducation religieuse et morale » (H. Lombaerts et E. Osewska) propose quelques grilles de lecture (systémique, philosophique) permettant de cerner les interpellations qui affectent les familles chrétiennes actuellement. Un deuxième texte « la catéchèse et l'éducation religieuse familiale dans l'Europe du $21^{\mathrm{e}}$ siècle » $(\mathrm{H}$. Lombaerts et E. Osewska) s'arrête davantage à la diversité qui s'installe tant au niveau ecclésial qu'en ce qui concerne la pratique catéchétique. Les auteurs montrent à quel point cette multiplicité induit différents modèles en vogue dans l'éducation religieuse familiale. Le troisième texte " l'éducation religieuse et la catéchèse dans la famille : une perspective psychologique et ecclésiale » (J. Stala) s'arrête au statut des enfants dans la société moderne et insiste sur l'importance de l'éducation religieuse familiale pour y faire face.

Une première partie présente les perspectives de sept pays de l'Europe Centrale et de l'Est: Lituanie, République Chèque, Slovaquie, Hongrie, Bulgarie, Slovène, Pologne. Une deuxième partie inclut des contributions de cinq pays 
de l'Europe Occidentale : Irlande, Allemagne, Belgique, Angleterre et Pays de Galles, Portugal.

Toutes les contributions font appel à des données sociologiques ou à des études empiriques pour décrire (l'évolution de) la situation de la famille dans la société contemporaine. Ces données permettent d'en identifier les variables sociales (République Chèque), ou de préciser l'influence des différentes instances impliquées dans l'éducation religieuse (O. Stefanak, Slovaquie). Les changements dus à la transition du système communiste vers la société occidentale, après 1989, semblent affecter directement les conditions de vie des familles et invitent à préciser le rôle d'une catéchèse familiale (E. Osewska/J. Stala, Pologne; J. Fogassy, Hongrie) ou à concevoir une pastorale d'accompagnement des couples et des familles (A. Segula, Slovène). La situation de la Bulgarie (importance de l'Eglise Orthodoxe Orientale, présence de l'Islam) crée des visées et pratiques spécifiques en ce qui concerne le rôle de la famille (B. Todorova, J. Donkova et R. Mudrov). Les deux articles qui présentent des recherches au sens propre du terme permettent d'observer de près l'importance de la transition. En ce qui concerne la Lituanie, les auteures en explorent l'impact sur la foi des différentes générations et leur positionnement par rapport à l'Eglise. Un nouveau dilemme semble émerger : le choix entre le relativisme et l'engagement religieux (B. Briliute et A. Karaliute). En ce qui concerne l'importance de la famille pour l'initiation chrétienne en Pologne, une étude comparée entre les pratiques d'initiation chrétienne dans les familles en 1986,1999 et 2009 montre l'impact incontestable de la transition de la société sur la religiosité de la famille et son rôle dans l'éducation chrétienne (E. Osewska).

Les auteurs représentant des pays de l'Europe Centrale et de l'Est, emploient souvent le mot « transition » pour identifier l'impact du changement de système socio-économique et idéologique sur les familles et leur rôle dans l'initiation chrétienne des enfants. Le mot « transition » est aussi utilisé dans les contributions venant des pays de l'Europe Occidentale. Ici il renvoie surtout à la mutation du contexte de la société occidentale pendant la deuxième moitié du $20^{\text {ème }}$ siècle, causant une implosion du christianisme centraliste et uniforme, avec des conséquences immédiates en ce qui concerne la nature et la structure des familles, et le rôle des parents dans l'éducation religieuse des enfants. Les différentes contributions s'appuient amplement sur les investigations sociologiques pour l'illustrer de manière convaincante. Cette évolution a surpris l'Irlande, un des pays les plus catholiques d'Europe, ce qui a fait apparaître l'absence d'une pastorale d'ensemble pour accompagner les parents dans leur mission dans ce nouveau contexte (P. Kieran). Ce souci d'une coordination entre les différents 
acteurs impliqués dans la formation religieuse au sein de la famille est davantage développée en Allemagne grâce aux livres conçus spécifiquement pour les parents (A. Biesinger et S. Hiller). L'éducation religieuse dans le contexte familial a été examinée de manière systématique et empirique en Flandres (Belgique) en interrogeant des jeunes à propos de leur expérience personnelle d'une initiation chrétienne en famille pendant leur enfance. Les données ainsi rassemblées permettent de préciser différentes manières dont l'Eglise peut aider les parents à s'impliquer davantage dans l'éducation religieuse de leurs enfants (A. Dillen). Bien que de nombreux documents sur l'éducation chrétienne aient inspiré et stimulé le développement d'une vision dynamique de l'initiation religieuse, il s'avère que la pratique renouvelée sur le terrain est profondément marquée par les conditions de vie concrète des parents et attend toujours une coordination d'ensemble plus cohérente (E. Davies, Angleterre et Pays de Galles). Tout en admettant que la famille a un rôle irremplaçable pour l'éducation des enfants, il s'impose qu'elle soit d'abord appréciée et valorisée davantage dans le contexte de la société contemporaine (civile) en vue d'aider les parents à mieux comprendre et assumer leur responsabilité dans l'éducation religieuse de leurs enfants (C. Sà Carvalho, Portugal).

Cette publication est importante car elle répond à un double souci : revaloriser l'importance de la famille dans l'éducation chrétienne des enfants, surtout dans le contexte de la société actuelle ; et amorcer un dialogue documenté, à plusieurs reprises fondé sur une recherche empirique approfondie, entre l'Europe Centrale et de l'Est et l'Europe Occidentale concernant cette catéchèse familiale. Tous les auteurs considèrent la famille comme lieu privilégié de l'initiation chrétienne, même si la responsabilité de la formation religieuse est progressivement et parfois exclusivement confiée à l'école et la paroisse.

En donnant la parole à des représentants des pays de la nouvelle Europe, les éditeurs rejoignent les nombreux efforts entrepris déjà pour dépasser les cloisonnements historiques dus à l'opposition entre deux systèmes qui ont marqué l'Europe durant presqu'un siècle. Ce conflit historique, et l'isolement qui l'a accompagné a marqué la manière de comprendre et de pratiquer l'initiation religieuse en famille, au risque d'aboutir à des visées entièrement différentes, voire antithétiques. Ce livre contribue de manière décisive à évacuer ce risque. Les Eglises des deux côtés de l'ancien « rideau de fer » ont été interpellées à se renouveler en réponse aux changements incontournables. Depuis le début des années 1990 les jeunes générations des pays de l'Europe Centrale et de l'Est se sont empressées de s'approprier les valeurs et les aspirations du monde occidental. Devant ce brassage d'influences et d'interactions multiples, les parents et les 
catéchètes s'interrogent sur l'impact de ces évolutions sur leurs efforts, ayant souvent l'impression de ne plus contrôler les composantes dynamiques de ce qui se présente comme une mutation.

Le titre du livre suggère qu'il s'agit d'une « perspective Européenne ». Plutôt, le lecteur y trouve une juxtaposition d'une série de perspectives présentées telles qu'elles existent dans les différents pays. Le poids de l'histoire propre à chaque pays oriente la perspective énoncée. La réception et l'interprétation des changements socio-économiques, politiques et culturelles guident les prises de position dans le domaine religieux, dans l'éducation religieuse et la catéchèse familiale en particulier.

Sans aucun doute, les différentes contributions permettent au lecteur de pratiquer un nouveau type de dialogue. Il est invité à repérer les préoccupations communes, les différences, les incompréhensions et l'impossibilité éventuelle d'établir un discours commun sur le thème proposé. L'intention de fond n'étant peut-être pas d'unifier et d'uniformiser les deux mondes vers une pensée et une pratique uniques, mais de découvrir à quel point et dans quel sens la diversité est indispensable pour re-créer et solidifier une identité chrétienne dans l'Europe de l'avenir. La responsabilité de se mettre à dialoguer est entièrement laissée au lecteur. 